\title{
ELECTROSTATICALLY-ACTUATED RECONFIGURABLE ELASTOMER MICROFLUIDICS
}

Meng-Ping Chang ${ }^{1}$, and Michel M. Maharbiz ${ }^{2,3}$

${ }^{1}$ Department of Mechanical Engineering, University of Michigan, Ann Arbor, USA

${ }^{2}$ Department of Electrical Engineering and Computer Science, University of California, Berkeley, USA

${ }^{3}$ Department of Electrical Engineering and Computer Science, University of Michigan, Ann Arbor, USA

\section{ABSTRACT}

We present a user-programmable reconfigurable elastomer microfluidic system which employs electrostatic actuation of water-filled elastomer microfluidic channels. Device actuation was achieved by applying $5 \mathrm{MHz}, 15-20 \mathrm{~V}$ voltages to induce "wet" electrostatic gap-closing of nano-liter PDMS microfluidic chambers embedded with metal flexure electrodes. The primary contributions of this technology are: a) elastomer microfluidics that do not require external pneumatics to actuate when filled with air, oil, or water, b) a fabrication process compatible with standard PDMS microfluidics, and c) actuation voltages low enough to be driven by off-the-shelf RF IC's.

\section{INTRODUCTION}

For the past fifteen years, microfluidics has been a research field of intense scientific and engineering interest [1]. Technologies based on elastomers such as polydimethylsiloxane (PDMS) and parylene have become very popular due to ease of fabrication and use [2, 3, 4]. Most these technologies require external pneumatic connections for liquid control, but this manipulation becomes problematic as the system advances toward high-density large-scale integration. The lack of local low power actuation also prevents in vivo applications of these devices [5].

In this paper, we present a completely reconfigurable distributed elastomer microfluidic network system, as shown in Fig. 1. The operation of the device and two designs of the fabricated chips are shown in Fig. 2. The device consists of 7 (hexagon) or 9 (square) micro chambers which can be actuated independently by electrostatic pull-in of a PDMS-metal membrane [6]. The embedded metal flexure forms the top capacitor plate and the ITO electrode forms the bottom plate. When a voltage above the pull-in voltage is applied, the PDMS-metal "roof” collapses onto the "floor" (Fig. 2 a) and liquid within this actuated chamber is expelled into adjacent open micro chambers. This applied signal had a $5 \mathrm{MHz}$ frequency to prevent electrical double layer screening of the electrostatic forces [7]. This makes possible the design of VLSI fluidic systems with many actuated components each driven by digitally synthesized signals. The device can actuate in air, water, and oil without external fluidic connections. These micro devices are also scalable to nanofluidic regimes and are compatible with general PDMS microfluidics.

\section{THEORY AND DEVICE DESIGN}

A key issue of designing this micro device is the pull-in voltage which is affected by the device geometry, mechanical properties and dielectric constants of the membranes and working liquids. A closed form of the pull-in voltage can be expressed as $[7,8]$ :

$$
V_{P I}=\sqrt{\frac{8 k\left(g+t_{o x} \varepsilon_{L} / \varepsilon_{o x}\right)}{27 \varepsilon_{0} \varepsilon_{L} A} \cdot\left(1+\frac{2\left(1-v^{2}\right) \sigma_{R} A}{9 E t^{2}}\right)}
$$

where $k=$ spring constant; tox $=$ oxide thickness; $g=$ channel height; $\varepsilon_{\mathrm{ox}}=$ oxide permittivity; $\sigma_{\mathrm{R}}=$ membrane residual

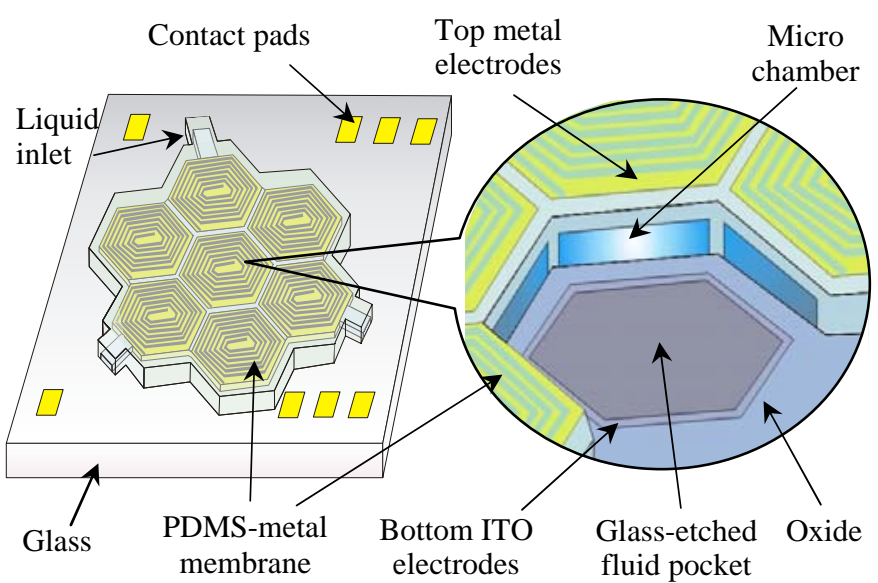

Figure 1: Conceptual view of a reconfigurable microfluidic device built on a glass substrate. The top PDMS-metal membrane and the bottom ITO form the capacitor plates. Each valve chamber is connected to adjacent chambers and can be electrostatically actuated independently. A valve seat is made by glass wet etching. An insulating oxide layer is deposited on the ITO electrode.

stress, $A=$ capacitor area; $\varepsilon \mathrm{L}=$ liquid dielectric permittivity; $v$ $=$ Poisson's ratio; $E=$ Young's modulus, and $t=$ membrane thickness.

Devices with square elements measured $300 \mu \mathrm{m} \times 300 \mu \mathrm{m}$ and hexagonal elements had $200 \mu \mathrm{m}$ edges, as shown in Fig. $2 \mathrm{~b}$. Hexagonal chambers were found preferable due to 1) compact footprint 2) shorter liquid transport path and 3) more uniform membrane deflection. The patterned spiral metal line was only for electric conduction of the PDMS, and its stiffness could be neglected when compared to that of PDMS membrane. The spring constant [9] of the devices and the pull-in voltage were calculated to be $20-30 \mathrm{~N} / \mathrm{m}$ and $15-20 \mathrm{~V}$. We previously characterized the pull-in behavior of this type of device [6]. The inlets connecting to each micro chamber were $50 \mu \mathrm{m}$ in width and $5 \mathrm{~mm}$ in length.

Table 1: Parameter values used in equation (1).

\begin{tabular}{||l|l||}
\hline \hline Square area & $9 \times 10^{4} \mu \mathrm{m}^{2}$ \\
\hline Hexagon area & $1 \times 10^{4} \mu \mathrm{m}^{2}$ \\
\hline Channel height gap & $2.5-5.0 \mu \mathrm{m}$ \\
\hline Oxide permittivity & 3.9 \\
\hline Oxide thickness & $0.4 \mu \mathrm{m}$ \\
\hline Liquid permittivity & 78.0 \\
\hline Membrane Young's modulus & $370.0 \mathrm{MPa}[12]$ \\
\hline Membrane residual stress & $6.4 \mathrm{MPa}[12]$ \\
\hline Membrane Poisson's ratio & $4.8[12]$ \\
\hline Membrane thickness & $7.2 \mu \mathrm{m}$ \\
\hline
\end{tabular}


(a)

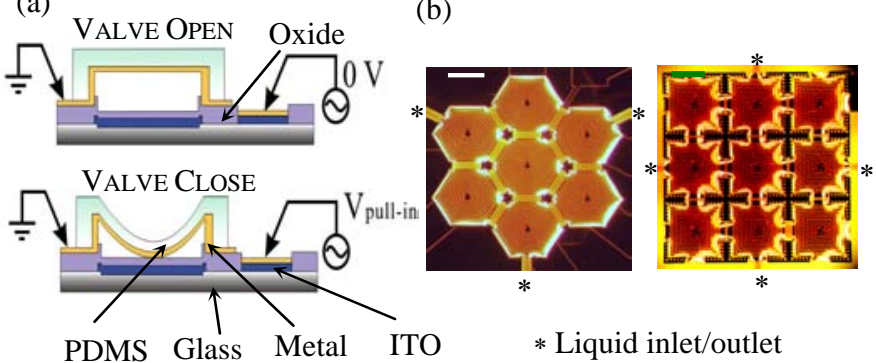

Figure 2: (a) The valve is closed when applying a $5 \mathrm{MHz}$ signal at the pull-in amplitude (15-20 volt). By varying the sequence of actuation signal the chambers can function as independent micro channels, valves, and pumps. (b) White-light micrograph of fabricated devices. Scale bar, $200 \mu \mathrm{m}$.

\section{FABRICATION}

Figure 3 shows the fabrication flow of a basic single valve. The glass substrate was first wet etched in diluted buffered HF to form a $1 \mu \mathrm{m}$ recess. Indium tin oxide (ITO, $1000 \AA$ thickness) was patterned, annealed at $700^{\circ} \mathrm{C}$, and covered with a layer of $4000 \AA$ plasma-enhanced chemical vapor deposition (PECVD) oxide. This oxide layer was annealed at $550^{\circ} \mathrm{C}$ for better dielectric insulation. Photoresist (AZ-9260, $10 \mu \mathrm{m}$ ) was then spun, baked and patterned into a micro channel mold. A thin film of $\mathrm{Cr} / \mathrm{Au}(150 / 5000 \AA)$ was then sputtered and patterned using wet-etching to form the top capacitor plate. A stiff PDMS (Dow Corning WL-5351) was then spun and cured to form the micro chambers and channels. After releasing in acetone and methanol, the final channel height and the thickness of PDMS-metal membrane were 2.5 - $4 \mu \mathrm{m}$ and $7.2 \mu \mathrm{m}$, respectively. The device was mount and wire bonded onto a PCB board.

\section{EXPERIMENTAL}

\section{Microscopy and Fluidic Characterization}

Figure 4 (a) shows the experiment apparatus of flow measurement by fluorescent or white-light microscopy on a Nikon TE-2000 inverted microscope. Food color and fluorescent dyes (fluorescein and ruthenium tris(bipyridine) chloride) were used as (a) Glass etch

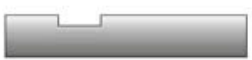

(d) Oxide Etch

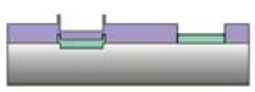

(g) Metal etch

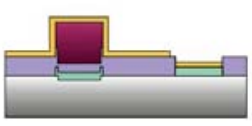

Figure 3: Fabrication flow.

visual indicators of valving actuation, flow movement and liquids mixing. Surfactant (Triton X-100) was mixed with water into $1 \%$ $\mathrm{w} / \mathrm{w}$ and filled the device by capillary force. Polystyrene microspheres (PolySciences, $0.5 \mu \mathrm{m}$ diameter) were mixed in the solution for flow rate measurement. A syringe pump (KD Scientific) was used to flush and fill the fluidic network. The counter pressure could be generated by a nitrogen bottle or hydrostatic pressure for pressure and flow rate characterization. Two pressure transducers (Honeywell 40PC-150) were installed on both stream sides of the device to measure pressure drop across the device.

\section{Control Circuitry}

The control circuitry is shown in Fig. 4 (b). A single valve device was actuated by a $5 \mathrm{MHz}$ signal, switched between $15-20 \mathrm{~V}$ amplitude and ground. An initial $5 \mathrm{MHz}, 5 \mathrm{Vp}-\mathrm{p}$ signal was generated by a function/arbitrary waveform generator (Agilent 33250A, 80MHz), and amplified to 35-40 Vp-p by either high speed IC amplifiers (Analog Devices AD815) or commercial RF amplifiers (Amplifier Research). The drive signal was applied via a pair of photovoltaic relay IC's (International Rectifier PVA13N) each of which was controlled by two digital signals $\left(P_{i}=\bar{P}_{i+1}\right)$ from the micro controller (Parallax BS2). The micro controller was programmed to provide the various actuation sequences used. (a)

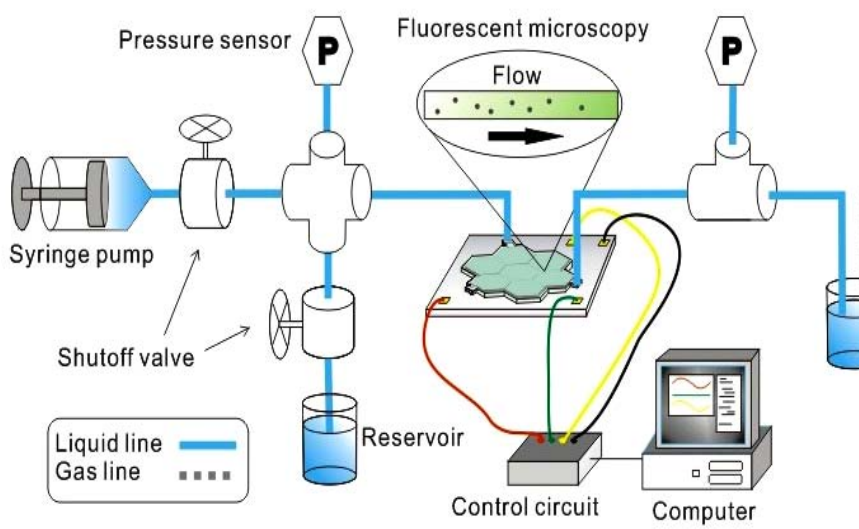

Figure 4: Experiment apparatus. (a) A syringe pump is connected at the upstream to flush/fill the system prior to any test. Two pressure transducers are installed to measure the pressure difference across the chip. A nitrogen bottle is used as pressure source to generate backpressure for pressure characterization. The flow velocity is measured by detecting the displacement of $0.5 \mu \mathrm{m}$ microspheres. (b) Control circuitry. Photovoltaic relay is controlled by a signal from the BS2 microcontroller. Signals from two adjacent ports $\mathrm{Pi}$ and $\mathrm{P} i+1$ in BS2 are always out of phase, so the $5 \mathrm{MHz}$ signal can be periodically switched between 15-20 V and ground. (b)

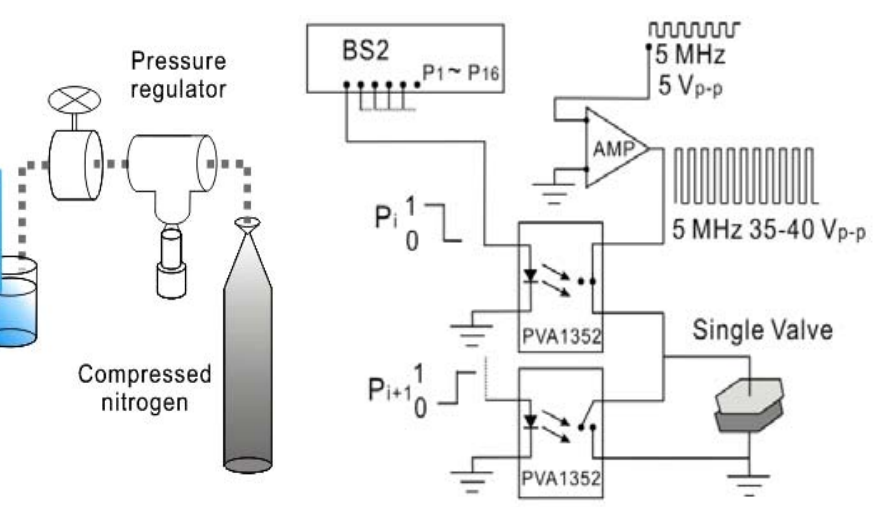


(a)

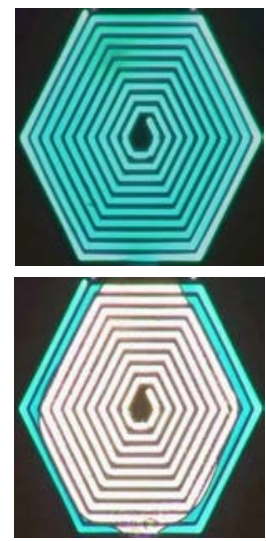

(b)

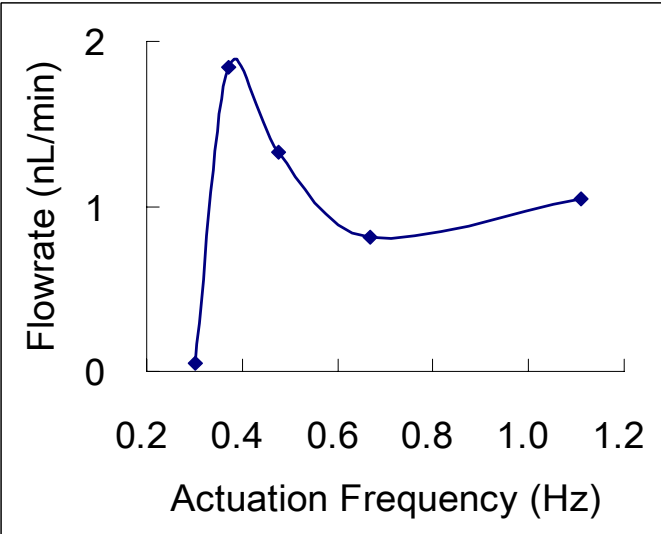

(c)

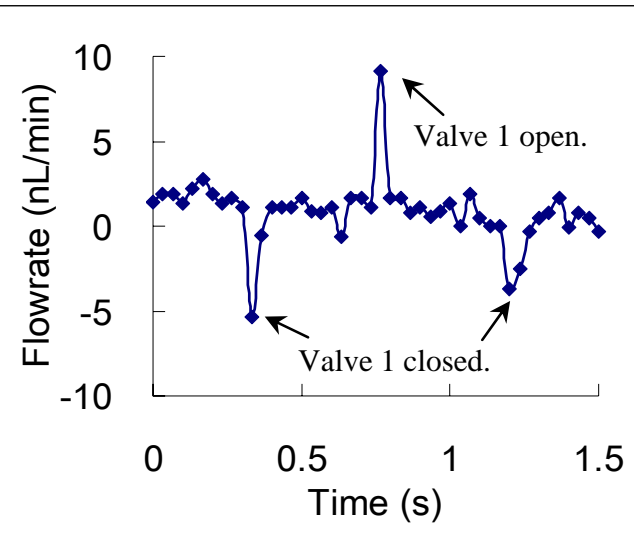

Figure 5: Valving and peristaltic pumping. (a) Top: an open valve filled with blue liquid. Bottom: as the valve closed, the liquid was expelled. (b) Average flow rate versus pumping frequency. The maximum average flow rate was found at $0.4 \mathrm{~Hz}$ actuation. After this

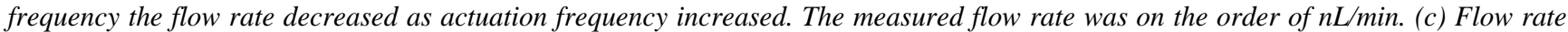
versus time with $1.11 \mathrm{~Hz}$ actuation frequency. Peristaltic pumping generated pulsating flow, as indicated by the positive and negative spikes in the plot. The span between the two negative spikes has the same time span as the actuation period (0.9 second).

\section{RESULTS AND DISCUSSION}

Single Valve

Figure 5 (a) shows the basic valve operation. An earlier design of square units was reported to hold a 6 psi pressure [10]. The peak current was less than $1 \mu \mathrm{A}$.

\section{Peristaltic Pump}

Figure 5 (b) shows the average flow rate versus actuation frequency of a three-valve peristaltic pump. The maximum flow rate is 4.45 valve volume/min $(1.85 \mathrm{~nL} / \mathrm{min})$ at $0.37 \mathrm{~Hz}$. After this frequency the flow rate decreased as frequency increased. Figure 5 (c) presents the time response of the pump flow rate at $1.1 \mathrm{~Hz}$ actuation frequency. The liquid movement was a pulsating flow, with a nominal flow rate about $2 \mathrm{~nL} / \mathrm{min}$. As expected, positive and negative velocity peaks were observed at the same frequency as the pump actuation frequency.

\section{Reconfigurable Distributed Microfluidics}

Table 2 shows the different actuation patterns used for valving, pumping and mixing. Figure 6 shows single-valve circulation actuation using a ruthenium tris(bipyridine) chloride dye as a fluorescent indicator. The actuation time of a single element was approximately $150 \mathrm{~ms}$. We also achieved bi-valve circulation, full vertical, full horizontal, "Y" pumping and mixing; all these videos are available online [11].

Fluid switching, as shown in Fig. 7, was realized by peristaltically actuating any three adjacent valves and closing all other undesignated valves. Blue food color was used to indicate flow direction.

Figure 8 shows mixing of two liquids. Two liquids, yellow and blue, were induced into the device from two separate liquid inlets. By actuating two pairs of diagonal valves sequentially, the liquids were actively mixed into green liquid. The whole mixing process was accomplished within 1 second.

\section{CONCLUSIONS}

We have designed, fabricated, and tested an electrostatic reconfigurable distributed microfluidic device. This technology is compatible with modern PDMS microfluidics and CMOS drive voltages. The device requires no external pneumatic connections, operates at low voltage and is conceptually scalable to nanofluidic regime. We have demonstrated the device is capable of single valving, peristaltic pumping, and multi-position serial valving, "Y" pumping, fluid switching, and mixing, all of which are essential liquid movement manipulations for large-scale parallel biological or biochemical analysis. Current work focuses on eliminating leakage by etching valve seats similar to those used in pneumatically-actuated valves [13].

Table 2: Device operation and actuation patterns.

\begin{tabular}{|l|l|l|}
\hline \\
valves 1, 3, and 5. Valve \\
number notation will be \\
used in Figures 5 - 8.
\end{tabular}



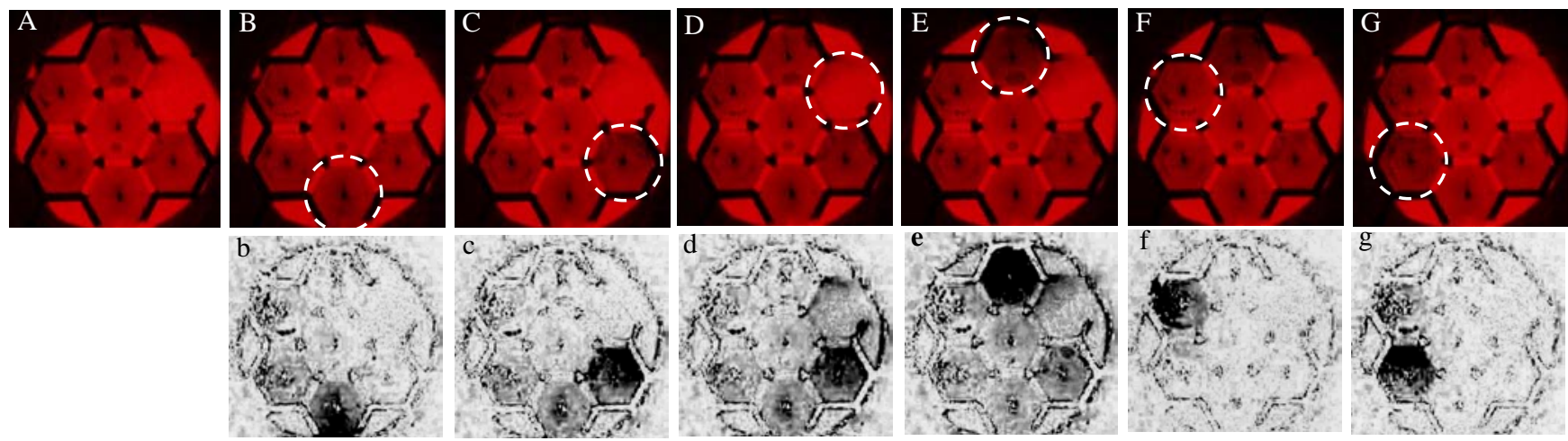

Figure 6: Single valve circulation actuation. Fluorescent microscopy images: image (A) is an unactuated device, and images B-G show independent actuation of each valve. White dashed circles indicate the actuated valve. Actuation voltage was $40 \mathrm{Vp}-\mathrm{p}$, and each individual actuation was $150 \mathrm{~ms}$. The motion of fluorophore is more apparent when images are subtracted from the background (A).
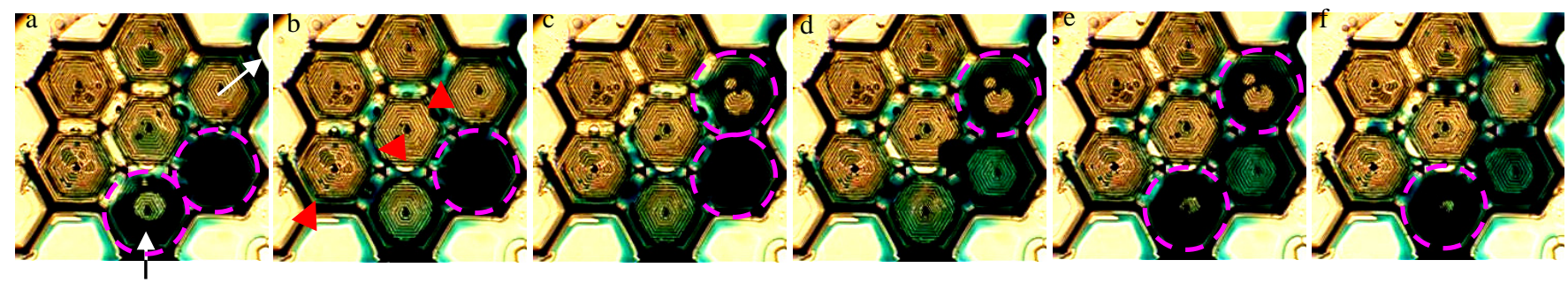

Figure 7: Fluid switching to the right. Liquid was pumped from valve 1 to valve 3, as indicated by the arrows in a). Valves 4, 5, 6, 7 were permanently closed, and valves 1,2 , 3 were actuated in sequence: $1+2 \rightarrow 2 \rightarrow 2+3 \rightarrow 3 \rightarrow 1+3 \rightarrow 1$. Dashed circles show the actuated valve for that phase of the peristaltic pumping. Each actuation period was $150 \mathrm{~ms}$, pumping frequency was $1.1 \mathrm{~Hz}$. Slight leakage (red arrowhead in b) occurred between the actuated chambers.
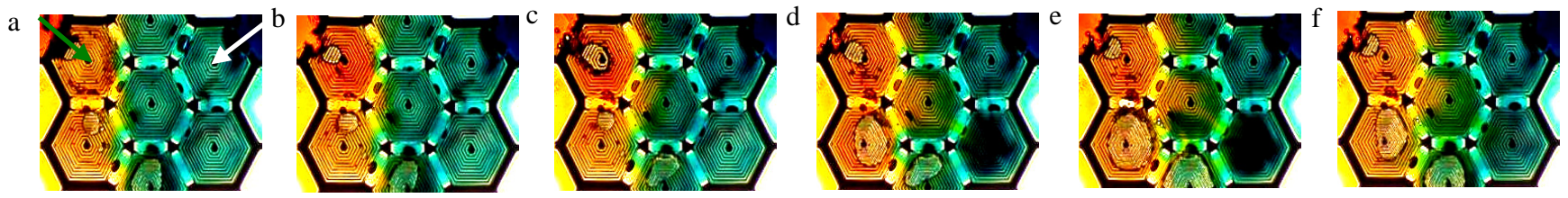

Figure 8: Mixing of two liquids in central chamber (valve 5). Time interval between each frame is $200 \mathrm{~ms}$. When $t=0$ (a), the chip was prefilled with blue liquid (valve 3, white arrow) and yellow liquid (valve 5, green arrow). At $t=200 \mathrm{~ms}$, valve 5 actuated and yellow liquid in valve 5 was expelled and started to mix with blue liquid in the central valve. This semi-mixed liquid was pushed into the lower-right valve (valve 2, d). As valve 2 actuated ( $t=800 \mathrm{~ms}$, fig. e) liquid was pushed back into valve 7, and completed one mixing cycle.

\section{ACKNOWLEDGEMENT}

The authors thank the Michigan Nanofabrication Facility, Neural Engineering Laboratory, Wise/Najafi Laboratory for experiment setup, Dr. Hirotaka Sato and Ruba Borno for discussion, and the funding support from the Engineering Research Centers Program of the National Science Foundation (EEC-9986866).

\section{REFERENCES}

[1] A. Manz, S. J. Harrison, E. M. J. Verpoorte, J. C. Fettinger, A. Paulua, H. Ludi, and H. M. Widmer, "Planar Chips Technology for Miniaturization and Integration of Separation Techniques into Monitoring Systems : Capillary Electrophoresis on a Chip”, J. Chromatogr., A, 593 (1992).

[2] M. A. Unger, H. P. Chou, T. Thorsen, A. Scherer, and S. R. Quake, "Monolithic Microfabricated Valves and Pumps by Multilayer Soft Lithography”, Science, 288 (2000).

[3] W. H. Grover, A. M. Skelley, C. N. Liu, E. T. Lagally, and R. A. Mathies, "Monolithic membrane valves and diaphragm pumps for practical large-scale integration into glass microfluidic devices”, Sens. Actuators, B, 89, 3 (2003).

[4] J. Xie, J. Shih, Q. Lin, B. Yang, and Y.-C. Tai, "Surface Micromachined Electrostatically Actuated Micro Peristaltic Pump”, Lab Chip, 4 (2004).

[5] N. A. Cellar, R. T. Kennedy, “A Capillary-PDMS Hybrid Chip for Separations-Based Sensing of Neurotransmitters in vivo", Lab Chip, 6 (2006).

[6] T. Bansal, M.-P. Chang, and M. M. Maharbiz, "A Class of Low Voltage, Elastomer-Metal "Wet" Actuators for Use in High-Density microfluidics", Lab Chip, 7 (2007).

[7] B. Legrand, A. S. Rollier, D. Collard, L. Buchaillot, "Suppression of the Pull-in Instability for Parallel-Plate Electrostatic Actuators Operated in Dielectric Liquids”, Appl. Phys. Lett., 88 (2006).

[8] P.-C. Hsu, C. H. Mastrangelo, K. D. Wise, “A High Sensitivity Polysilicon Diaphragm Condenser Microphone”, Proceedings of 1998 MEMS, 01//1998, Heidelberg, Germany (1998), pp. $580-585$.

[9] W. C. Young, "Roark's Formulas for Stress and Strain”, McGraw-Hill International Edition, (1989).

[10] M.-P. Chang, T. Bansal, and M. M. Maharbiz, "Electrically Actuated PDMS Microvalves and Pumps for VLSI Microfluidics" The 11th $\mu$ TAS, 10//2007, Paris, France (2007).

[11] http://www-personal.umich.edu/ mpchang/HH2008/

[12] Dow Corning WL-5351 product information.

[13] W. H. Grover, R. H. C. Ivester, E. C. Jensen, and R. A. Mathies, "Development and multiplexed control of latching pneumatic valves using microfluidic logical structures", Lab Chip, 6 (2006). 